AIRWAY BIOLOGY

\title{
Rapid and efficient clearance of airway tissue granulocytes through transepithelial migration
}

\author{
J S Erjefält, L Uller, M Malm-Erjefält, C G Persson
}

Thorax 2004;59:136-143. doi: 10.1136/thorax.2003.004218

See end of article for authors' affiliations

.....................

Correspondence to: Dr J S Erjefält, Department of Physiological Sciences, BMC F10, Lund University Hospital, 22184 Lund, Sweden; jonas.erjefalt@ mphy.lu.se

Received 17 January 2003 Accepted 16 October 2003
Background: Clearance of tissue granulocytes is central to the resolution of airway inflammation. To date the focus has been on apoptotic mechanisms of cell removal and little attention has been given to alternative processes. The present study explores transepithelial migration as a mechanism of cell clearance.

Method: Guinea pig tracheobronchial airways where eosinophils are constitutively present in the mucosal tissue were studied. A complex topical stimulus (allergen challenge) was applied and the fate of the eosinophils was determined by selective tracheobronchial lavage and histological examination of the tissue.

Results: Within 10 minutes of the allergen challenge, massive migration of eosinophils into the airway lumen occurred together with a reduction in tissue eosinophil numbers. Cell clearance into the lumen continued at high speed and by 30 and 60 minutes the tissue eosinophilia had been reduced by $63 \%$ and $73 \%$, respectively. The marked transepithelial migration (estimated maximal speed $35000 \mathrm{cell} / \mathrm{s} / \mathrm{min} \times$ $\mathrm{cm}^{2}$ mucosal surface) took place ubiquitously between epithelial cells without affecting epithelial integrity as assessed by transmission and scanning electron microscopy. Eosinophil apoptosis was not detected but occasional cytolytic eosinophils occurred.

Conclusion: This study shows that luminal entry has a remarkably high capacity as a granulocyte elimination process. The data also suggest that an appropriate stimulus of transepithelial migration may be used therapeutically to increase the resolution of inflammatory conditions of airway tissues.
T he airway mucosal defence system is dependent on its remarkable capacity to rapidly recruit and host cells of the immune system. However, to avoid a large accumulation of leucocytes, cell recruitment must be balanced by effective mechanisms for clearance of immune cells from the tissue. This is especially true for potentially tissue damaging effector cells such as eosinophil granulocytes. During allergic disorders such as allergic rhinitis and asthma, eosinophil accumulation occurs in the nasal and tracheobronchial airways. While detailed information is available on processes involved in eosinophil recruitment into airway tissues, less is known regarding the elimination of these cells from airway tissue in vivo.

Studies of the clearance of granulocytes from tissues to date have focused on elimination by apoptosis, a programmed cell death at the molecular level. ${ }^{1-4}$ It is currently believed that apoptosis, followed by prompt engulfment by neighbouring macrophages, is the mechanism by which airway tissue granulocytes are cleared. ${ }^{34}$ The fact that purified blood granulocytes spontaneously undergo apoptosis under standard incubation conditions in vitros has provided ample opportunities for detailed study of the molecular biology of apoptosis. ${ }^{6-8}$ However, the importance of granulocyte apoptosis in the tracheobronchial airway tissue in vivo has not been established, and significant eosinophil apoptosis in airway tissues has not yet been convincingly demonstrated in vivo, ${ }^{9}$ nor has apoptosis been compared with other potential modes of cell clearance from mucosa lined organs.

Based on new in vivo data and new interpretations of many previous observations, we recently proposed that transepithelial migration, in addition to being a mucosal defence mechanism, also offers a powerful potentially noninjurious alternative to apoptosis for effective clearance of airway tissue granulocytes. ${ }^{9}{ }^{10}$ In support of this hypothesis, it was recently reported that pharmacological attenuation of the transepithelial migration of eosinophils in allergic mice by neutralisation of MMP2 leads to a marked accumulation of these cells in the tissue. ${ }^{11}$ Observations on luminal samples have shown that transepithelial migration of leucocytes occurs during many conditions of airway inflammation. ${ }^{12-16}$ It is also clear that migration into the lumen inevitably leads to a corresponding reduction in the number of cells in the tissue. Yet, this pathway has so far largely been ignored as a potential clearance route for mucosal granulocytes. ${ }^{9}$

The aim of the present study was to explore the kinetic capacity of transepithelial migration to resolve an established airway tissue eosinophilia in vivo and to determine the nature of this mode of cell clearance. For this purpose we needed a model with a stable baseline eosinophilia where the quantitative assessment of eosinophil movement was not complicated by de novo recruitment of eosinophils to the tissue of interest. To our knowledge, the only such model is guinea pig large extrapulmonary airways which are characterised by a natural mucosal eosinophilia. ${ }^{17}{ }^{18}$ In preliminary experiments using this model and sensitised animals we observed that mucosal challenges such as allergen exposure appeared to reduce the numbers of eosinophils present in the tissue under baseline conditions. ${ }^{18}{ }^{19}$ Using the acute luminal entry of resident tissue eosinophils and complementary histological analyses of both tissue sections and tracheal whole mount preparations, we determined the speed by which transepithelial migration may be induced in vivo. We also examined whether the reduction in tissue eosinophilia by transepithelial migration caused injury to the epithelial surface. The occurrence of other eosinophil elimination processes such as apoptosis ${ }^{1}$ and cytolysis ${ }^{20}$ has also been studied to compare the different modes of eosinophil clearance. 


\section{METHODS \\ Sensitisation and allergen challenge of the tracheobronchial airways}

Male guinea pigs (Dunkin-Hartley, 200 g, Möllegaard, Denmark) were sensitised to ovalbumin (OVA) by one intraperitonal injection of $0.5 \mathrm{ml}$ saline containing $100 \mathrm{mg}$ $\mathrm{Al}(\mathrm{OH})_{3}$ and $1 \mu \mathrm{g}$ OVA. ${ }^{21}$ Three weeks after sensitisation animals were challenged by local allergen exposure of the tracheobronchial airways as previously described. ${ }^{19}$ Briefly, animals were anaesthetised by an intramuscular injection $(1 \mathrm{ml} / \mathrm{kg})$ of a $2: 3$ ratio of xylazine $(20 \mathrm{mg} / \mathrm{kg})$ and ketamine $(50 \mathrm{mg} / \mathrm{kg}$ ) and placed in an upright supine position. A thin plastic catheter was gently inserted via the mouth into the tracheal lumen $5 \mathrm{~mm}$ below the larynx. Using a microinjector (Carnegie Medical, Sweden) and a pump rate of $20 \mu \mathrm{l} / \mathrm{min}$, the allergen ( 10 pmol OVA in saline) was administered to the upper airways over a 2 minute period (control animals received saline only). Importantly, this technique evenly and selectively distributes the allergen to the mucosal surface of the large airways only-that is, the trachea and main bronchi. ${ }^{21}$ The experiment was terminated 2, 10, 30, and 60 minutes after initiation of the allergen challenge when luminal and tissue samples were collected for further analysis. At each time point 10 animals were analysed (four saline and six OVA challenged).

\section{Eosinophils in tracheobronchial lavage fluid}

A previously validated technique was used to collect upper airway surface liquid without disturbing baseline mucosal barrier functions. ${ }^{21}$ Immediately after the animals were killed the airways (including larynx and lungs) were excised totally. The lung lobes were tied off at the hilar region and a small incision was made in the left mainstream bronchus. A catheter (PE50) was introduced, secured, and the airways were mounted vertically. The upper airway was then rinsed by pushing $0.25 \mathrm{ml}$ saline through the trachea and the adjoining main bronchi three times. Lavage samples were obtained as described above and $10 \mu \mathrm{l}$ was used to determine the total cell content using a haemocytometer. 20000 cells were applied to glass slides using a cytospin centrifuge (Shandon Cytospin 3, UK) and eosinophil numbers cell were determined after staining with May-Grunewald Giemsa. The luminal eosinophil count was expressed as total numbers/ $0.2 \mathrm{ml}$ lavage fluid.

\section{Tissue granulocytes}

Two $4 \mathrm{~mm}$ tracheal rings separated by $5 \mathrm{~mm}$ were immersed in fixative $(4 \%$ paraformaldehyde in $0.1 \mathrm{M}$ phosphate buffered saline (PBS), $\mathrm{pH} 7.2$ ) overnight at $4^{\circ} \mathrm{C}$. After rinsing in Tyrode buffer (PBS buffer supplemented with 10\% sucrose), the rings were frozen on mounting stubs using Tissue Tec mounting medium. Eosinophils in the tracheal mucosa were detected in $10 \mu \mathrm{m}$ transversally cut cross sections by histochemical staining of cyanide resistant eosinophil peroxidase. ${ }^{18}$ Tissue sections were incubated at room temperature for 8 minutes in a PBS buffer supplemented with 3,3-diaminobenzidine tetrahydrochloride $(75 \mathrm{mg} /$ $100 \mathrm{ml}), 30 \% \mathrm{H}_{2} \mathrm{O}_{2}(0.3 \mathrm{ml} / 100 \mathrm{ml})$, and sodium cyanide $(50 \mathrm{mg} / 100 \mathrm{ml})$. The sections were then counterstained and mounted in Kaiser's medium (Merck, Germany). The numbers of intraepithelial and subepithelial tissue eosinophils (eosinophils present in the subepithelial tissue down to the perichondrial layer of the tracheal cartilage) were then quantified at $\times 200$ magnification in two separate tracheal regions/animal and expressed as cells/tracheal cross section area (in separate experiments it was shown that the variation in the analysed tissue section area between individual samples was small enough not to affect the quantification).
In separate sections neutrophils were visualised by staining for neutrophil endogenous peroxidase. Briefly, sections were rinsed in Tris buffer and incubated at room temperature for 5 minutes in a Tris $\mathrm{HCl}$ buffer $(\mathrm{pH} \mathrm{9.0)}$ containing $0.1 \%$ naphthol AS-BI phosphate (Sigma) and $0.1 \%$ Fast Blue (TRsalt, Sigma). The neutrophils were easily identified by their blue staining and characteristic polymorph nuclei. ${ }^{18}$ The total number of neutrophils (from the apical surface of the airway epithelium down to the perichondrial layer of the tracheal cartilage) in each section was quantified and expressed as cells/tracheal cross section.

\section{Assessment of eosinophils in tracheal whole mount preparations}

At the end of the experiment the trachea was cut along its dorsal side, gently stretched out on Sylgard coated petri dishes, and immediately placed in fixative $(4 \%$ paraformaldehyde in 0.1 M PBS, pH 7.2) overnight at $4^{\circ} \mathrm{C}$. For proper orientation the apical surface of the airway epithelium was visualised by histochemical staining of tissue non-specific alkaline phosphatase (TNAP) as previously described. ${ }^{19}$ Whole mount preparations were incubated at room temperature for 5 minutes in a Tris $\mathrm{HCl}$ buffer $(\mathrm{pH}$ 9.0) containing $0.1 \%$ naphthol AS-BI phosphate (Sigma) and $0.1 \%$ Fast Blue (TR-salt, Sigma). TNAP is expressed in the apical membrane of columnar epithelial cells and in separate control experiments it was shown in cross section whole mount preparations that the TNAP staining was restricted to the apical membrane of the epithelium. After rinsing in Tris buffer the preparations were stained with eosinophil peroxidase (EPO) as described above. The whole mount preparations were then mounted in Kaiser's medium and examined under a bright field microscope. Eosinophils were identified as distinct dark brown spheres. The three dimensional distribution of eosinophils was determined using the X-Y scale (lateral distribution) and focal scale (depth) of the microscope. The surface epithelium (dotted blue) and basement membrane (identified by the appearance of extracellular fibres) were used as depth reference points. The difference in the whole mount preparation content of eosinophils at baseline $(n=6)$ and 10 minutes after OVA challenge $(n=6)$ was used to obtain an estimation of the total numbers of eosinophils leaving the tissue during the first acute migration. Eosinophils were quantified at $200 \times$ magnification by counting the numbers of EPO positive cells in eight focal planes.

The occurrence of eosinophil cytolysis (ECL) was also quantified in the whole mount preparations using the presence of clusters of free eosinophil granules as positive selection criteria. ${ }^{20}$ ECL was also analysed by transmission electron microscopy. At an ultrastructural level, ECL was identified by chromatolysis and ruptured cell membranes leading to an extracellular distribution of cell organelles. ${ }^{20}$

\section{Assessment of apoptosis \\ TUNEL staining}

Cryosections were allowed to dry at room temperature and then treated with proteinase $\mathrm{K}(20 \mu \mathrm{g} / \mathrm{ml}$, Sigma, Sweden $)$ for 15 minutes at room temperature. Apoptotic cells in airway tissue were identified using a commercial TUNEL technique kit (ApoTag Fluoroscein, Intergen Company, NY, USA). ${ }^{102}$ Thymus from steroid treated rats was used as a positive control (occurrence of green fluoroscent cell nuclei in apoptotic cells). Staining was absent in negative controls-that is, when the Tdt enzyme was omitted. Apoptotic cells were defined as cells displaying TUNEL positive nuclei and otherwise apoptotic morphology such as condensed chromatin and cytoplasm.9 ${ }^{22}$ TUNEL stained sections were counterstained with the DNA binding dye 
Hoechst 3332 to reveal the nuclear morphology of all cells stained. Finally, eosinophils were visualised by EPO staining as described above.

\section{Transmission electron microscopy}

Intact viable eosinophils were identified by their characteristic granules, normal chromatin pattern in the cell nucleus, and preserved integrity of the cytoplasmatic and nuclear membranes. Apoptotic eosinophils were defined as cells with dark condensed nuclear chromatin, organelle swelling, and preserved cell membranes. ${ }^{9}$ Transmission electron microscopy was also used to identify any phagocytic cells containing apoptotic cells or cell debris within phagosomes.

\section{Integrity of airway epithelium}

The integrity of the airway epithelium during the first hour after challenge was assessed by two complementary techniques. Scanning electron microscopy was used to get an overview of the epithelial surface structure and to analyse the structure of the apical epithelial cell borders ${ }^{23}$ and the occurrence of any potential allergen induced patchy epithelial damage. ${ }^{24}$ The detailed structure of epithelial integrity, including the appearance of tight junctions and desmosomes in the apical portion of the epithelium, was studied by transmission electron microscopy. ${ }^{23} 24$

\section{Scanning electron microscopy}

A segment of the trachea was cut along the ventral side, stretched out on a Sylgard coated petri dish, and immersed in electron microscopy fixative (PBS buffer supplemented with $1 \%$ glutaraldehyde and 3\% formaldehyde). After fixation overnight, samples were rinsed thoroughly in PBS, dehydrated through a series of increasing ethanol solutions, and subjected to critical point drying using $\mathrm{CO}_{2}$ as the transitional fluid. ${ }^{24}$ The specimens were then coated with $15 \mathrm{~nm}$ gold and examined using a Philips 515 scanning electron microscope (Philips, The Netherlands).

\section{Transmission electron microscopy}

After fixation in PBS buffer supplemented with $1 \%$ glutaraldehyde and 3\% formaldehyde overnight, the tracheal samples were rinsed in buffer, post-fixed in $1 \%$ osmium tetroxide for 1 hour, and dehydrated in graded acetone solutions and embedded in Polarbed 812. One $\mu \mathrm{m}$ thick plastic sections were examined by bright field microscopy and areas with a well preserved morphology were selected for electron microscopic analysis. Ultrathin sections $(90 \mathrm{~nm})$ were cut and placed on a 200 mesh thin bar copper grid before staining with uranyl acetate and lead citrate. The specimens were examined using a Philips CM-10 transmission electron microscope (Philips, The Netherlands).

\section{Statistical analysis}

All data in the figures are expressed as mean (SE). The data were $\log$ transformed to achieve normal distribution before statistical analysis. For the main results, the effect of treatment was also presented as the quotient of OVA versus saline treatment with 95\% confidence intervals (95\% CI).

The significance of the overall differences between treatments and between time points was assessed by analysis of variance (ANOVA) according to Tukey. The difference in treatments between each time point was assessed by Student's $t$ test. The statistical analysis was performed using Astute 1.5, a statistics add-in for Microsoft Excel (DDU Software, Leeds, UK); p values of $<0.05$ were considered statistically significant.

\section{RESULTS}

\section{Allergen challenge induced acute clearance of mucosal tissue eosinophils}

Abundant mucosal eosinophils were present under baseline conditions-that is, in naive control animals-and in sensitised but not OVA exposed controls (figs 1 and 2). Within 10 minutes of the challenge in sensitised animals there was a significant fall in total tissue eosinophils compared with saline exposed controls (fig 2C, table 1). Subanalysis of intraepithelial and subepithelial eosinophils at this time point revealed a significant reduction in epithelial eosinophils while the number of subepithelial eosinophils remained unaltered (fig $2 \mathrm{~A}$ and $\mathrm{B}$ ). A further reduction in total tissue eosinophils was observed at 30 and 60 minutes after challenge (fig 1A). At these time points a reduction in both intraepithelial and subepithelial eosinophils was present (figs 1 and 2, table 1).

To estimate the numbers of eosinophils leaving the tissue during the acute transepithelial migration phase, the total eosinophil content was determined at baseline and $10 \mathrm{~min}$ utes after OVA challenge. A statistical difference $(p<0.001)$ was found between the eosinophil content in tracheal tissue collected at baseline ( $1.02(95 \%$ CI 0.91 to 1.12$) \times 10^{6}$ cells/ $\mathrm{cm}^{2}$ mucosal surface) and that collected 10 minutes after OVA challenge $(0.67$ (95\% CI 0.62 to 0.71$) \times 10^{6}$ cells $/ \mathrm{cm}^{2}$ mucosal surface). From the known fall in tissue eosinophila
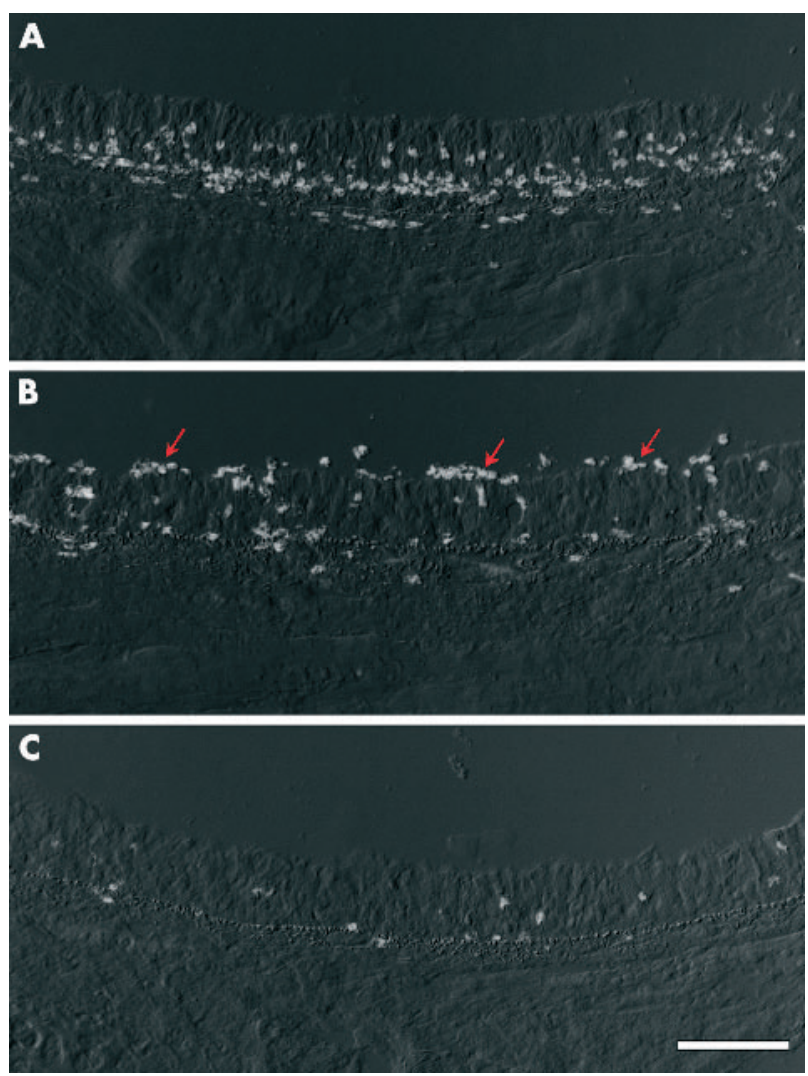

Figure 1 Histological images showing (A) EPO stained eosinophils in the tracheal mucosa under baseline conditions. Note the dense distribution of eosinophils in the basal part of the airway epithelium and in the nearby subepithelial tissue. (B) Thirty minutes after the challenge the number of eosinophils in the tissue is reduced and abundant eosinophils can be seen on the luminal surface (red arrows). (C) At 60 minutes a further reduction in eosinophilia is evident in both the epithelial and subepithelial tissue compartments. The EPO-reaction complex (white precipitates) was visualised by epipolarisation microscopy and the contour of the non-stained section with Normarsky optics. Bar $=120 \mu \mathrm{m}$. 

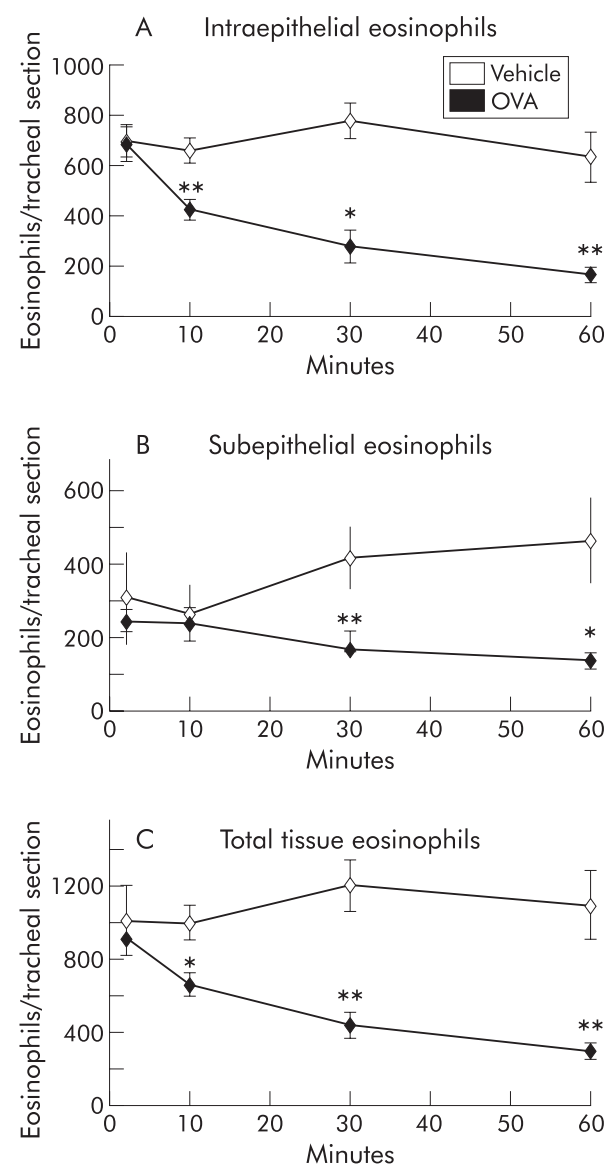

Figure 2 Numbers of (A) intraepithelial, (B) subepithelial, and (C) total tissue eosinophils in the tracheal wall during the first hour after allergen exposure. All data are presented as mean (SE) eosinophils per tracheal cross section. ${ }^{*} p<0.05,{ }^{* *} p<0.01$ differences between vehicle (PBS) and ovalbumin (OVA) challenged animals.

it could be estimated that, during the first 10 minutes after challenge, $0.35 \times 10^{6}$ eosinophils had left each $\mathrm{cm}^{2}$ of the mucosal surface.

\section{Numbers of eosinophils in airway lumen}

Ten minutes after the challenge the numbers of eosinophils in the tracheobronchial lavage samples were significantly increased compared with saline treated controls (tables 1 and $2)$. The numbers of luminal eosinophils were further increased at 30 and 60 minutes.

The luminal influx of eosinophils was confirmed by analysis of tracheal whole mount preparations using EPO histochemistry (fig $3 \mathrm{~A}$ and $\mathrm{B}$ ) and scanning electron microscopy (fig $3 \mathrm{C}$ and D). At baseline and in saline treated controls only a few scattered eosinophils were detected at the mucosal surface. In contrast, 10 minutes after challenge and subsequently, abundant eosinophils had accumulated on the mucosal surface (fig 3).

\section{Assessment of epithelial integrity}

Scanning electron microscopy did not detect any changes in epithelial surface morphology 10 and 30 minutes after the challenge. At 60 minutes no change was detected in the epithelial structure apart from a patchy occurrence of focal sites of ongoing epithelial damage restitution-that is, the occurrence of undifferentiated epithelial repair cells. ${ }^{24}$ The area affected by this change was $<0.25 \%$ of the total epithelial surface. Transmission electron microscopy con-
Table 1 Effect of allergen challenge on clearance of eosinophils from airway tissue

\begin{tabular}{|c|c|c|c|}
\hline & Time (min) & Effect of challenge $†$ & $\mathrm{p}$ value \\
\hline Intraepithelial & 2 & 1.0 (0.7 to 1.4$)$ & \\
\hline \multirow[t]{3}{*}{ eosinophils } & 10 & $0.6(0.5$ to 0.9$)$ ** & \\
\hline & 30 & $0.3(0.1$ to 0.5$)$ ** & \\
\hline & 60 & $0.2(0.1$ to 0.5$)$ ** & $<0.0001$ \\
\hline Subepithelial & 2 & $0.9(0.4$ to 1.9$)$ & \\
\hline \multirow[t]{3}{*}{ eosinophils } & 10 & $0.9(0.4$ to 2.0$)$ & \\
\hline & 30 & $0.4(0.2 \text { to } 0.7)^{* *}$ & \\
\hline & 60 & $0.2(0.1 \text { to } 0.4)^{* * *}$ & 0.0013 \\
\hline Total tissue & 2 & $0.9(0.6$ to 1.4$)$ & \\
\hline \multirow[t]{3}{*}{ eosinophils } & 10 & $0.7(0.5$ to 0.9$)$ * & \\
\hline & 30 & $0.3(0.2$ to 0.5$)$ ** & \\
\hline & 60 & $0.2(0.1 \text { to } 0.4)^{* * *}$ & $<0.0001$ \\
\hline \multirow{4}{*}{$\begin{array}{l}\text { Lavage content of } \\
\text { eosinophils }\end{array}$} & 2 & $0.8(0.2$ to 3.6$)$ & \\
\hline & 10 & $7.0(1.1$ to 46$)$ * & \\
\hline & 30 & $12(1.5 \text { to } 108)^{*}$ & \\
\hline & 60 & $32(3.3 \text { to } 314)^{\text {** }}$ & 0.0004 \\
\hline \multirow[t]{4}{*}{ Eosinophil cytolysis } & 2 & $0.9(0.5$ to 1.5$)$ & \\
\hline & 10 & $1.0(0.5$ to 3.6$)$ & \\
\hline & 30 & $3.6(1.6 \text { to } 8.0)^{* *}$ & \\
\hline & 60 & $5.9(2.8 \text { to } 12)^{* *}$ & $<0.0001$ \\
\hline \multirow[t]{4}{*}{ Neutrophils } & 2 & $4.6(1.4 \text { to } 15)^{*}$ & \\
\hline & 10 & $22(5.6 \text { to } 86)^{* *}$ & \\
\hline & 30 & $20(3.8 \text { to } 107)^{\star *}$ & \\
\hline & 60 & $36(8.3 \text { to } 158)^{* * *}$ & 0.0008 \\
\hline \multicolumn{4}{|c|}{$\begin{array}{l}\dagger \text { Effect of challenge expressed as the quotient of ovalbumin (OVA) } v \\
\text { saline treatment }(95 \% \mathrm{Cl}) \text {. } \\
\ddagger \text { The significance of difference between treatments and time points } \\
\text { overall. } \\
{ }^{*} p<0.05,{ }^{* *} p<0.01,{ }^{* * *} p<0.001 \text {. }\end{array}$} \\
\hline
\end{tabular}

firmed that the epithelial integrity was preserved and revealed a normal structure of the tight junction and desmosomes in areas subjected to intensive transepithelial eosinophil migration.

\section{Occurrence of eosinophil cytolysis (ECL) acutely after allergen challenge}

Cytolysis of eosinophils examined by identification of clusters of free eosinophil granules in tracheal whole mount preparations, was rarely present in the eosinophils of control animals or of animals examined 2 and 10 minutes after allergen challenge (fig 4). An increase in the proportion of eosinophils undergoing ECL was observed 60 minutes after the challenge (figs 4 and 5, table 1). Tissue depth analysis of cytolytic cells revealed that the distribution of ECL was mainly restricted to the vicinity of the epithelial basement membrane (fig 5). The presence of cytolytic eosinophils was confirmed by transmission electron microscopy which showed eosinophils in the subepithelial tissue just beneath the basement membrane displaying chromatolytic cell nuclei and ruptured cell membranes.

\section{Eosinophil apoptosis}

Identification of TUNEL positive cells revealed that, at baseline, apoptosis occurred only rarely in saline treated animals $\left(1.6(0.4)\right.$ cells $\left./ \mathrm{mm}^{2}\right)$. No significant increase in

Table 2 Tracheobronchial lavage content of eosinophils

\begin{tabular}{lll}
\hline Time & Saline controls & Ovalbumin challenged \\
\hline $2 \mathrm{~min}$ & $0.33(0.14)$ & $0.22(0.10)$ \\
$10 \mathrm{~min}$ & $0.11(0.09)$ & $0.70(0.31)$ \\
$30 \mathrm{~min}$ & $0.35(0.18)$ & $2.33(0.58)$ \\
$60 \mathrm{~min}$ & $0.10(0.07)$ & $3.62(0.77)$ \\
\hline
\end{tabular}

*All data are presented as mean (SE) cell numbers $\times 10^{6} / \mathrm{ml}$. 

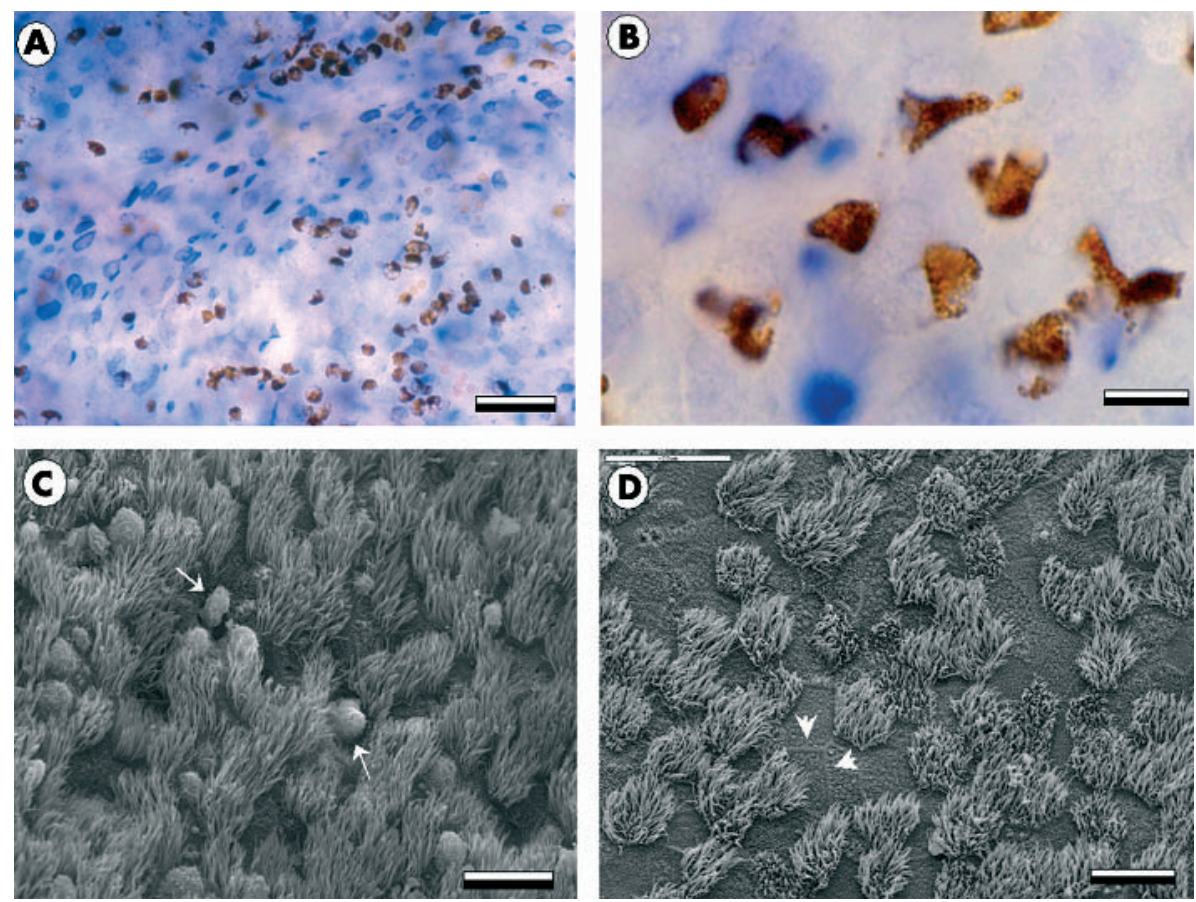

Figure 3 Micrographs showing the airway mucosa viewed from above in tracheal whole mount preparations. (A), (B) Bright field images in which eosinophils are identified by EPO histochemistry (brown) and the epithelial surface by TNAP staining (blue and white pattern). At 30 minutes numerous eosinophils can be seen at the epithelial surface (A, B). (C) At the same time point scanning electron microscopy reveals the ciliated epithelial surface and the presence of surface associated leucocytes (white arrows). (D) At 60 minutes the airway epithelium shows no signs of epithelial damage and displays normal tight seams where the apical membranes of neighbouring cells meet (arrow heads). Scale bars in $A=80 \mu \mathrm{m}, \mathrm{B}=10 \mu \mathrm{m}, \mathrm{C}=40 \mu \mathrm{m}$, and $\mathrm{D}=40 \mu \mathrm{m}$.

apoptosis was observed after allergen challenge when the total numbers of apoptotic cells at 30 and 60 minutes were 4.0 (2.0) and 3.2 (1.4) cells $/ \mathrm{mm}^{2}$, respectively.

Most of the TUNEL positive cells were of a non-eosinophil phenotype (lack of EPO staining in the cytoplasm, fig 5). Of all the cells examined, only one TUNEL positive eosinophil could be found in saline treated animals and one apoptotic eosinophil was detected after allergen challenge. The paucity of eosinophil apoptosis at the time points examined was confirmed by transmission electron microscopy which failed to detect any apoptotic eosinophils. Transmission electron microscopy also failed to detect apoptotic cells or cell debris inside the phagosomes of phagocytic cells. In total, only three large mononuclear cells containing phagosomes with cell debris were identified. In these cases the identity or cause of death of the engulfed cells could not be determined.

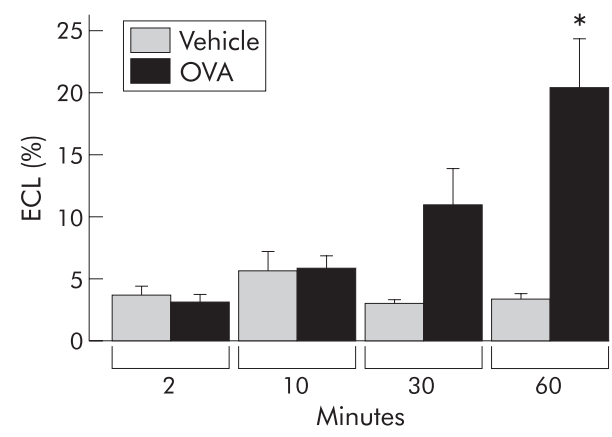

Figure 4 Proportion of tissue eosinophils undergoing cytolysis. Eosinophil cytolysis (ECL) was measured in tracheal whole mount preparations. ${ }^{18}$ All data are presented as mean (SE) eosinophils per tissue area unit. ${ }^{*} \mathrm{p}<0.05$ difference between vehicle (PBS) and ovalbumin (OVA) challenged animals.

\section{De novo recruitment of neutrophils and eosinophils}

Within 10 minutes of the challenge and thereafter the number of TNAP positive neutrophils in the tracheal tissue was significantly increased compared with PBS treated controls (fig 6, table 1). At 10 minutes the neutrophils were mainly distributed in the tracheal blood vessels whereas by 30 and 60 minutes they were present in both intravascular and extravascular compartments. Intravascular EPO stained eosinophils could not be detected at any of the time points examined.

\section{DISCUSSION}

This study shows that transepithelial migration into the airway lumen is a highly efficient mechanism for clearance of mucosal tissue eosinophils in vivo, with tissue eosinophilia being halved during the first 30 minutes after the application of a topical challenge agent. The speed of clearance is reflected by the finding that, on average, around 500 eosinophils cross each $\mathrm{cm}^{2}$ of the tracheal epithelium every second during the initial 10 minutes after challenge. We also found that the eosinophils migrated ubiquitously between columnar epithelial cells to enter the airway lumen without inflicting damage to the epithelial lining cells. This study confirms the occurrence of primary ECL. ${ }^{20}$ However, no apoptotic eosinophils were detected in the mucosal tissue, inside or outside of macrophages, indicating that the acute clearance of eosinophils into the airway lumen is not complemented by any other non-inflammatory mode of elimination of these cells. Luminal entry alone is therefore a highly efficient mode of non-injurious elimination of mucosal eosinophils in vivo.

Eosinophil clearance by apoptosis has received much attention and, as for neutrophils, apoptosis has generally been considered the only important clearance mechanism. ${ }^{9}$ The eosinophil pro-apoptosis actions of anti-asthma drugs 

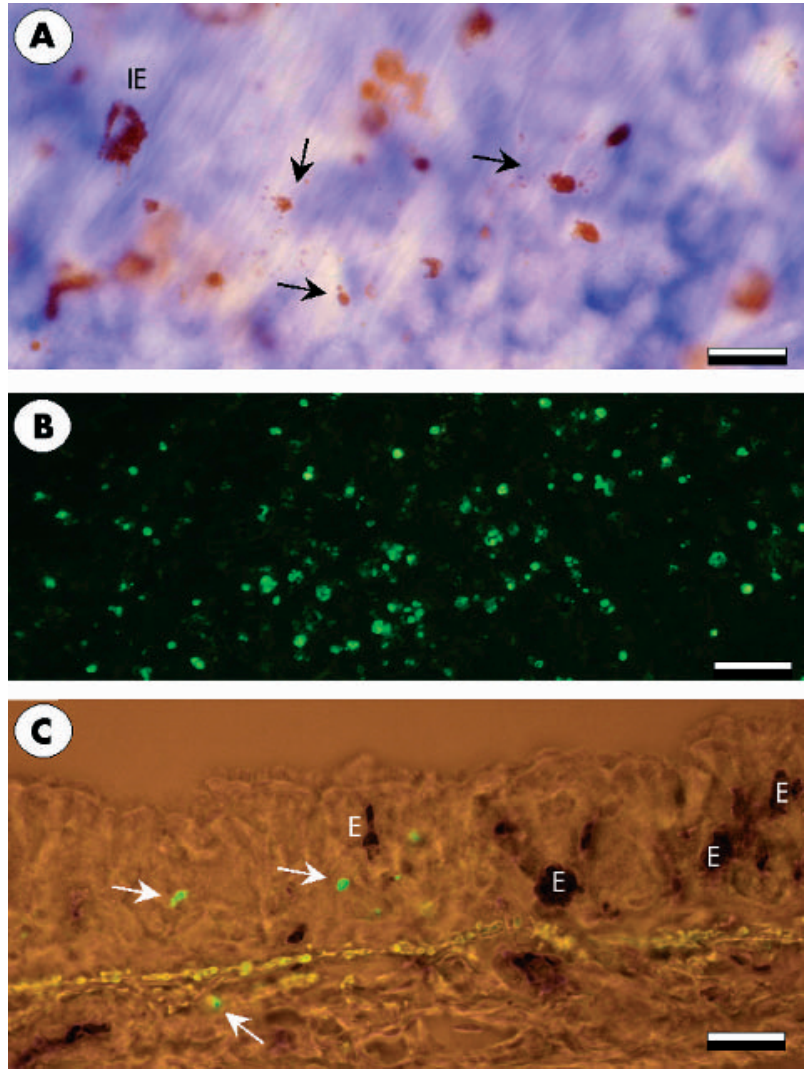

Figure 5 Whole mount tracheal preparations showing the occurrence of eosinophil cytolysis 60 minutes after allergen exposure. (A) Cytolytic cells are seen as clusters of free eosinophil granules (arrows). (B) Fluoroscence micrograph of abundant stained cells in thymus which was used as a positive control for TUNEL staining. (C) TUNEL positive cells of a non-eosinophil phenotype in the airway mucosa. $E=$ eosinophils. Bars: $\mathrm{A}=10 \mu \mathrm{m}, \mathrm{B}=80 \mu \mathrm{m}, \mathrm{C}=20 \mu \mathrm{m}$.

have also been considered to be highly significant. ${ }^{25} 26$ Since this interest in eosinophil apoptosis has not been matched by the finding of an in vivo role for this mechanism, ${ }^{9}$ we have looked at alternative modes of elimination of these cells from airway tissues. We therefore sought new information on some basic aspects of airway luminal entry of eosinophils including the kinetic capacity of this mechanism for cell clearance. An in vivo approach was essential. It was also necessary to have experimental conditions with a stable established eosinophilia to allow the disappearance of eosinophils to be studied without any confounding by de novo recruitment of these cells into the study area. We therefore used guinea pig large airways where the eosinophilia constitutively is of the order of that observed in human diseased allergic airways. ${ }^{18}$ In this model we also had access to well validated methods for controlled low traumatic exposure and lavage specifically of the area of interest. ${ }^{21}{ }^{27}$ This is important because airway eosinophilia is not present in guinea pig intrapulmonary airways, obviating the use of common inhalation and bronchoalveolar lavage techniques. The choice of allergen challenge in sensitised animals to effect the removal of airway tissue cells was based on our preliminary observations on the entry of eosinophils into the airway lumen during the first hour after acute allergic reactions in this model. ${ }^{19}$ As borne out by the present findings, this methodology was well suited for studying the features of acute luminal entry as a mode of cell clearance.

The use of baseline eosinophilia in the present study reveals, for the first time, the remarkable capacity by which

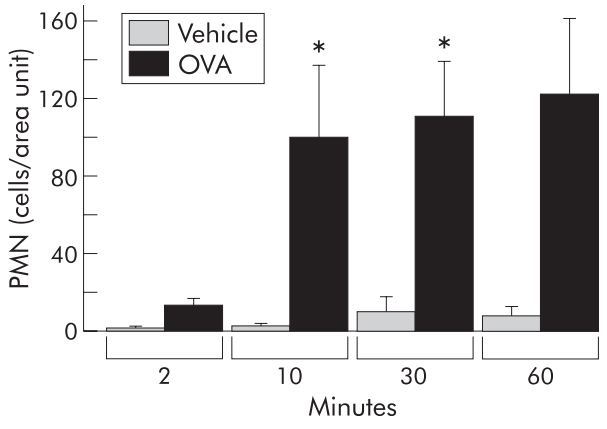

Figure 6 Numbers of neutrophils (PMN) in tracheal sections (total neutrophils per tissue area). All data are presented as mean (SE) neutrophils per tracheal cross section. ${ }^{*} p<0.05$ difference between vehicle (PBS) and ovalbumin (OVA) challenged animals.

transepithelial migration occurs in vivo. Histological examination showed that the tissue eosinophils were mobilised and migrated towards the mucosal surface minutes after topical allergen challenge. Thus, during the first 10 minutes after the challenge (when apoptosis and cytolysis were rare or absent) it was estimated that an average of around 350000 eosinophils had left the tissue across each $\mathrm{cm}^{2}$ of the mucosal surface. This cellular response therefore occurs as rapidly as the acute physiological responses to allergen challenge such as smooth muscle constriction and plasma extravasation. ${ }^{27} 28$ However, there is no reason to assume that transepithelial migration must be accompanied by other acute allergic airway actions (as discussed below, extensive transepithelial migration of eosinophils also occurs during other stages of eosinophilic inflammation). Despite the fact that no further stimulus was applied, the clearance continued so that by 30 minutes the number of tissue eosinophils had been halved. These data indicate that the luminal entry mechanism is an efficacious and speedy method of removing eosinophil granulocytes from the airway mucosa.

The fact that eosinophil apoptosis did not increase during the course of the study indicates that luminal entry by itself is a powerful mechanism by which bronchial mucosal eosinophils can be cleared in vivo. ${ }^{9-11}$ The role of egress into the airway lumen may, of course, not only be valid for the acute elimination of cells as studied here. Numerous human and animal studies have shown that the entry of mucosal eosinophils into the airway lumen occurs during late phase allergic reactions. ${ }^{15} 2930$ Similarly, during natural or drug induced resolution of an eosinophilic inflammation, eosinophils continuously leave the tissue by migration into the airway lumen. ${ }^{9}$ For example, recent experimental work focusing on slow resolution of airway tissue eosinophilia occurring over several days has shown that luminal entry is important while there was no evidence of eosinophil apoptosis. ${ }^{10}$ Furthermore, steroid induced resolution of eosinophilia occurred without any detectable eosinophil apoptosis. Steroid treatment, in addition to preventing de novo recruitment of eosinophils into the lung, may permit elimination of these inflammatory cells from airway tissues via the airway lumen. ${ }^{10}$

Despite the intense migration of cells across the epithelial lining, we found no detectable damage to the mucosal surface. This suggests that transepithelial migration occurs without affecting the integrity of the epithelium. ${ }^{31}$ Previous work has also shown that allergen challenge in this model does not cause increased paracellular epithelial permeability, determined as the rate of absorption of hydrophilic tracer molecules applied to the mucosal surface. ${ }^{32}$ The immediate plasma exudation response, as evoked by allergen challenge, therefore has no effect on absorption permeability, although 
it involves the luminal entry of non-sieved plasma macromolecules. ${ }^{33}$ The facilitation of non-injurious transepithelial passage of cells and extravasated molecules into the lumen thus seems to be a general feature of the airway epithelium. As expected, this function of the epithelial lining may serve both clearance and defence purposes. For example, the rapid transepithelial passage of granulocytes into the lumen means that these cells can be mobilised to neutralise infectious or other exogenous attacks on the surface before the target tissue has been penetrated by the offensive organism. ${ }^{34}{ }^{35}$

Eosinophil cytolysis is a primary mode of activationdegranulation ${ }^{20}$ which occurs in eosinophils showing no signs of apoptosis. In sensitive areas of the airway the spilling of protein laden granules that results from primary cytolysis may have a local effect on the epithelial lining. ${ }^{19}{ }^{20}$ ECL could therefore have caused some of the restricted focal damage observed which, in the present model, involved less than $0.2 \%$ of the mucosal surface. ${ }^{24}$ The observation that the proportion of ECL was increased only when the tissue eosinophilia had been dramatically attenuated also suggests that this mode of elimination had only a minor effect on reducing tissue eosinophils.

The focus of the present study is on early events occurring within the first half hour after challenging the mucosa. As expected, neutrophils (but not eosinophils) were observed adhering to the vascular endothelium, reflecting early stages of accumulation of these cells in the allergen challenged airways. We were therefore able to examine the movement of the eosinophils already in the tissues without the need to consider newly recruited eosinophils. It was also important to determine the eosinophils remaining in the tissue to avoid too much reliance on the lumen where physiological clearance mechanisms (such as mucociliary clearance) would make data less precise. The present method of superfusion, as well as the actions of the allergen, could potentially reduce baseline mucociliary clearance of luminal cells by impairing the function of cilia (beating in too much fluid). Mucociliary clearance nevertheless did occur since measurements carried out at 5 hours showed significantly reduced numbers of luminal eosinophils. This finding should be compared with an estimated mucociliary transport rate in the normal trachea of about $1 \mathrm{~cm} / \mathrm{min} .{ }^{36}$ Although no such movement was determined in this study, it cannot be excluded that some eosinophils may return into the airway tissue. ${ }^{37}$ However, it seems likely that the vast number of cells trapped in viscous luminal secretions/exudates would not be likely to re-enter the airway tissue but would be removed by mucociliary clearance and coughing.

Further studies are needed to address the mechanisms involved in clearance of eosinophils from airway tissue to determine the effect of specific migratory stimuli and the fate of the cells after they have entered the airway lumen. Whereas plasma exudation across an intact airway epithelium may be driven by small increments in basolateral epithelial hydrostatic pressure, ${ }^{33}$ the movement of leucocytes most likely involves chemoattractant molecules. In support of this notion, it has been shown that, when stimulated, epithelial cells can perform a polarised release of chemokines towards the lumen ${ }^{38}$ and that leucocytes may be driven across sheets of epithelial cells by chemokines apparently without reducing the tightness of the epithelial cell layer. ${ }^{39}$

This study has shown that transepithelial migration into the airway lumen represents an efficient mechanism for clearance of leucocytes from the mucosal tissue. When induced by an appropriate stimulus this mechanism can, in the absence of any significant apoptotic or other elimination mechanisms, promptly resolve an established tissue eosinophilia. The entry of eosinophils (and potentially other mucosal inflammatory cells) into the airway lumen is therefore a promising new area of research for studying the resolution of mucosal inflammation and for developing treatment strategies to resolve cellular airway inflammation.

\section{ACKNOWLEDGEMENTS}

This study was funded by the Medical Faculty, Lund, Sweden, the Vårdal Foundation, the Swedish Medical Research Council (VR K2002-74X 13407-03A), and the Swedish Heart and Lung Foundation.

\section{Authors' affiliations}

J S Erjefält, L Uller, Department of Physiological Sciences, Lund University, Lund, Sweden

M Malm-Eriefält, C G Persson, Department of Clinical Pharmacology, Lund University, Lund, Sweden

\section{REFERENCES}

1 Golstein P, Ojcius DM, Young JD. Cell death mechanisms and the immune system. Immunol Rev 1991;121:29-65.

2 Stern M, Meagher L, Savill J, et al. Apoptosis in human eosinophils. Programmed cell death in the eosinophil leads to phagocytosis by macrophages and is modulated by IL-5. J Immunol 1992;148:3543-9.

3 Simon HU, Blaser K. Inhibition of programmed eosinophil death: a key pathogenic event for eosinophilia? Immunol Today 1995; 16:53-5.

4 Bratton DL, Fadok VA. "Their's but to do and die": eosinophil longevity in asthma. J Allergy Clin Immunol 1999;103:555-8.

5 Yamaguchi Y, Suda T, Ohta S, et al. Analysis of the survival of mature human eosinophils: interleukin-5 prevents apoptosis in mature human eosinophils. Blood 1991; 78:2542-7.

6 Peachman KK, Lyles DS, Bass DA. Mitochondria in eosinophils: functional role in apoptosis but not respiration. Proc Natl Acad Sci USA 2001;98:1717-22.

7 Yousefi S, Green DR, Blaser K, et al. Protein-tyrosine phosphorylation regulates apoptosis in human eosinophils and neutrophils. Proc Natl Acad Sci USA 1994;91:10868-72.

8 Temple R, Allen E, Fordham J, et al. Microarray analysis of eosinophils reveals a number of candidate survival and apoptosis genes. Am J Respir Cell Mol Biol 2001:25:425-33

9 Erjefält JS, Persson CG. New aspects of degranulation and fates of airway mucosal eosinophils. Am J Respir Crit Care Med 2000;161:2074-85.

10 Uller L, Persson CG, Kallstrom L, et al. Lung tissue eosinophils may be cleared through luminal entry rather than apoptosis: effects of steroid treatment. Am J Respir Crit Care Med 2001;164:1948-56.

11 Corry DB, Rishi K, Kanellis J, et al. Decreased allergic lung inflammatory cell egression and increased susceptibility to asphyxiation in MMP2-deficiency. Nat Immunol 2002:3:347-53.

12 Keatings VM, Barnes PJ. Granulocyte activation markers in induced sputum: comparison between chronic obstructive pulmonary disease, asthma, and normal subjects. Am J Respir Crit Care Med 1997; 155:449-53.

13 Hansel $\pi$, Walker $C$. The migration of eosinophils into the sputum of asthmatics: the role of adhesion molecules. Clin Exp Allergy 1992;22:345-56

14 Huber FK, Koessler KK. The pathology of bronchial asthma. Arch Intern Med 1922;30:689-760.

15 Persson C GA. Centennial notions of asthma as an eosinophilic, desquamative, exudative, and steroid-sensitive disease. Lancet 1997;350:1021-4.

16 Diukanovic R, Roche WR, Wilson JW, et al. Mucosal inflammation in asthma Am Rev Respir Dis 1990;142:434-57.

17 Li D, Wang D, Griffiths-Johnson DA, et al. Eotaxin protein and gene expression in guinea-pig lungs: constitutive expression and upregulation after allergen challenge. Eur Respir J 1997;10:1946-54.

18 Erjefält JS, Sundler F, Persson CG. Eosinophils, neutrophils, and venular gaps in the airway mucosa at epithelial removal-restitution. Am J Respir Crit Care Med 1996; 153:1666-74

19 Erjefält JS, Korsgren M, Nilsson MC, et al. Association between inflammation and epithelial damage-restitution processes in allergic airways in vivo. Clin Exp Allergy 1997;27:1345-57.

20 Persson CG, Erjefält JS. Eosinophil lysis and free granules: an in vivo paradigm for cell activation and drug development. Trends Pharmacol Sci 1997:18:117-23.

21 Erjefält I, Greiff L, Alkner U, et al. Allergen-induced biphasic plasma exudation responses in guinea pig large airways. Am Rev Respir Dis 1993; 148:695-701.

22 Gavrieli $Y$, Sherman Y, Ben Sasson SA. Identification of programmed cell death in situ via specific labeling of nuclear DNA fragmentation. J Cell Biol 1992;119:493-501.

23 Erjefält JS, Erjefält I, Sundler F, et al. In vivo restitution of airway epithelium. Cell Tissue Res 1995;281:305-16.

24 Eriefält JS, Korsgren M, Nilsson MC, et al. Prompt epithelial damagerestitution processes in allergen-challenged guinea-pig trachea. Clin Exp Allergy 1997;27:1458-71.

25 Druilhe A, Wallaert B, Tsicopoulos A, et al. Apoptosis, proliferation, and expression of $\mathrm{Bcl}-2$, Fas, and Fas ligand in bronchial biopsies from asthmatics. Am J Respir Cell Mol Biol 1998;19:747-57. 
26 Adachi T, Motojima S, Hirata A, et al. Eosinophil apoptosis caused by theophylline, glucocorticoids, and macrolides after stimulation with IL-5. $J$ Allergy Clin Immunol 1996;98:S207-15.

27 Erjefält IA, Wagner ZG, Strand SE, et al. A method for studies of tracheobronchial microvascular permeability to macromolecules. J Pharmacol Methods 1985;14:275-83.

28 Bochner BS, Lichtenstein LM. Anaphylaxis. N Engl J Med 1991;324:1785-90.

29 Sedgwick JB, Calhoun WJ, Vrtis RF, et al. Comparison of airway and blood eosinophil function after in vivo antigen challenge. J Immunol 1992;149:3710-18.

30 Frigas E, Gleich GJ. The eosinophil and the pathophysiology of asthma. J Allergy Clin Immunol 1986;77:527-37.

31 Carolan EJ, Casale TB. Neutrophil transepithelial migration is dependent upon epithelial characteristics. Am J Respir Cell Mol Biol 1996; 15:224-31.

32 Erjefält I, Persson CG. Allergen, bradykinin, and capsaicin increase outward but not inward macromolecular permeability of guinea-pig tracheobronchial mucosa. Clin Exp Allergy 1991;21:217-24.
33 Persson CG, Eriefält I Alkner U et al. Plasma exudation as a first line respiratory mucosal defence. Clin Exp Allergy 1991;21:17-24.

34 Gewirtz AT, Siber AM, Madara JL, et al. Orchestration of neutrophil movement by intestinal epithelial cells in response to Salmonella typhimurium can be uncoupled from bacterial internalization. Infect Immun 1999;67:608-17.

35 Hang L, Frendeus B, Godaly G, et al. Interleukin-8 receptor knockout mice have subepithelial neutrophil entrapment and renal scarring following acute pyelonephritis. J Infect Dis 2000;182:1738-48.

36 Zhang P, Summer WR, Bagby GJ, et al. Innate immunity and pulmonary host defense. Immunol Rev 2000;173:39-51.

37 Shi HZ, Humbles A, Gerard C, et al. Lymph node trafficking and antigen presentation by endobronchial eosinophils. J Clin Invest 2000;105:945-53.

38 McCormick BA, Parkos CA, Colgan SP, et al. Apical secretion of a pathogenelicited epithelial chemoattractant activity in response to surface colonization of intestinal epithelia by Salmonella typhimurium. J Immunol 1998;160:455-66.

39 Milks LC, Brontoli MJ, Cramer EB. Epithelial permeability and the transepithelial migration of human neutrophils. J Cell Biol 1983;96:1241-7.

\section{LUNG ALERT}

Mesothelin-family proteins: a novel diagnostic marker for malignant mesothelioma? $\Delta$ Robinson BWS, Creaney J, Lake R, et al. Mesothelin-family proteins and diagnosis of mesothelioma. Lancet 2003;362:1612-6

$\mathrm{T}$

he pathological diagnosis of malignant mesothelioma (MM) remains difficult and special immunohistochemistry based stains are often required. This paper describes the role of mesothelin - a cell surface protein-and related soluble proteins in the diagnosis and monitoring of MM. The serum concentration of soluble mesothelin related proteins (SMR) was measured by sandwich ELISA in 44 patients with histologically proven MM, 68 (including 40 with history of exposure to asbestos) matched healthy controls, and 160 patients with inflammatory or malignant disorders of the lung and/or pleura.

37 of 44 patients with MM $(84 \%)$ had a raised concentration of SMR compared with asbestos exposed $(p=0.0003)$ and non-asbestos exposed controls $(p=0.0002)$. Seven of the 40 asbestos exposed controls had a raised level of SMR, three of whom developed mesothelioma within 1-5 years. Only three of the 160 patients with non-MM pleural/lung pathology (one each with cryptogenic fibrosing alveolitis, asbestosis, and NSCLC) had a raised concentration of SMR. None of the seven patients with lung adenocarcinoma had increased levels of SMR. The serum concentration of SMR at the time of diagnosis did not correlate with patient survival, but SMR concentrations correlated with tumour size and disease progression.

These data indicate that the concentration of SMR may be a sensitive early marker for the diagnosis of MM, particularly of the epithelioid variety, and may prove to be a useful screening tool in monitoring at risk individuals exposed to asbestos. Further studies are required to determine the prognostic importance of SMR and to determine the specificity of the assay in diagnosing mesothelioma.

A B Rajasekaran

Birmingham Heartlands Hospital, Birmingham, UK; arvind.rajasekaran@heartsol.wmids.nhs.uk 